\title{
Labor Pain Treated with Acupuncture or Acupressure
}

\author{
Oroma B. Nwanodi \\ Locum Tenen, Salinas, USA \\ Email: o.nwanodi@juno.com
}

How to cite this paper: Nwanodi, O.B. (2016) Labor Pain Treated with Acupuncture or Acupressure. Chinese Medicine, 7, 133-152.

http://dx.doi.org/10.4236/cm.2016.74014

Received: November 3, 2016

Accepted: November 27, 2016

Published: December 1, 2016

Copyright (@) 2016 by author and Scientific Research Publishing Inc. This work is licensed under the Creative Commons Attribution International License (CC BY 4.0).

http://creativecommons.org/licenses/by/4.0/

(c) (i) Open Access

\begin{abstract}
Opioid-dependent women have an $80 \%$ to $90 \%$ unintended pregnancy rate, almost double the overall unintended pregnancy rate: $40 \%$ globally and $51 \%$ in north America. The prescription drug abuse milieu increases the possibility opioid abusing laboring patients. In 2012, neonatal abstinence syndrome occurred in 5.8 per 1000 hospital births. Non-pharmacological labor pain management (NPLPM) is especially recommended for laboring patients with a history of substance abuse. Therefore, literature review was performed to elucidate the efficacy and safety of acupuncture, noninvasive electro-acupuncture (EA), and acupressure in labor pain management. Compared to standard intrapartum controls, bilateral EA at Jiajin or Sanyinjiao significantly reduced visual analog scale (VAS) pain scores 30-minutes post intervention $(\mathrm{p}<0.01)$ and Stage 1 active phase labor length $(\mathrm{p}<0.05)$. EA achieves shorter Stage 2 labor than patient-controlled epidural analgesia $(\mathrm{p}=0.05)$; and 10-point lower VAS pain scores and reduced cesarean delivery rate than no-analgesia controls, $\mathrm{p}<0.05$. Current evidence indicates that EA should have a role in NPLPM, and that acupressure may have a role in NPLPM. Nevertheless, future RCTs could strengthen the argument for increased EA and acupressure use in NPLPM.
\end{abstract}

\section{Keywords}

Acupuncture, Acupressure, Cesarean Delivery, Complementary Therapies, Electro-Acupuncture, Labor Pain Treatment, Manual Acupuncture, Non-Pharmacological Labor Pain Management, Obstetrics

\section{Introduction}

This article is a clinical review article for obstetricians, midwives, labor attendants, obstetric nurses, and doulas on the efficacy of acupuncture and acupressure treatment of labor pain. Labor pain management (LPM) is an integral part of women's labor expe- 
rience [1]. Uncontrolled labor pain is associated with vascular spasm, uncoordinated uterine contractions, paradoxically prolonged labor, and fetal hypoxia [2]. Therefore, uncontrolled labor pain should be avoided.

Currently, the prescription drug abuse milieu increases the possibility that a laboring patient has a history of opioid abuse [3]. In the United States, from 2002-2004 to 2011-2013, past-year heroin use increased $62.5 \%$ to 2.6 per 1000 persons 12 years old, with 18 to 25 year olds having a 108.6\% increase [4]. By 2009, 4.5\% of American households with pregnant women reported illegal drug use [5]. Among pregnant women using substance abuse treatment facilities, opioid abuse increased from $2 \%$ in 1992 , to $28 \%$ in 2012 [6]. Concurrently, in the United States, neonatal abstinence syndrome admissions to neonatal intensive care units (NICUs) almost tripled, increasing from 7 per 1000 in 2004 to 27 per 1000 in 2013 [7]. This is consistent with the 2012 incidence of neonatal abstinence syndrome in 5.8 per 1000 hospital births [8]. Given an $80 \%$ to $90 \%$ unintended pregnancy rate, opioid-dependent women have almost double the overall rate of unintended pregnancies: $40 \%$ globally and 51\% in north-America [5] [9]. Laboring patients with a history of substance abuse do experience genuine labor pain and can receive regional analgesia [10]. However, non-pharmacological labor pain management (NPLPM) should be used in laboring patients with a history of substance abuse [11].

Globally, women are interested in NPLPM. A cross-sectional study in south-eastern Nigeria showed that $59.6 \%$ of antepartum patients were interested in NPLPM [12]. In the United States only $21 \%$ to $46 \%$ of pregnant women may be aware of NPLPM choices [13]. When NPLPM is used, user ratings of somewhat effective or very effective can reach $72 \%$ to $100 \%$ [13]. Obstetricians, midwives, labor attendants, obstetric nurses, and doulas may not have an interest in alternative, complementary, holistic, or integrated medicine that promotes NPLPM. However, pregnant women are interested in NPLPM and movements are underway to bring these treatments under conventional insurance coverage, increasing access and demand [14] [15] [16].

The purpose of this review article is to elucidate the efficacy of acupuncture and acupressure in LPM. Secondary goals are identification if acupuncture, electro-acupuncture (EA), and acupressure have fewer adverse effects than epidural analgesia, and if women receiving acupuncture or acupressure LPM are less likely to undergo cesarean delivery than women who do not receive acupuncture or acupressure labor pain management. Recent randomized controlled trials (RCT) of acupuncture, EA, and acupressure will be reviewed. The efficacy of acupuncture, EA, and acupressure will be presented. Where possible, the adverse effects of these analgesic modalities, and any effect on cesarean delivery $(\mathrm{CD})$ rate will be presented.

\subsection{Terminology Definition}

Acupuncture is needle penetration at acupoints for analgesic effect. Manual acupuncture (MA) is a traditional technique with post-placement acupuncture needle rotation. Electro-acupuncture involves application of high and/or low frequency current to inva- 
sive or noninvasive acupuncture needles. Acupoint location is described using "cun" for fine needling Traditional Chinese Medicine (TCM) acupuncture, but for non-invasive EA and acupressure metric measurements are used for larger device positioning [17] [18]. Acupressure is pressure application instead of needle penetration at acupoints for analgesic effect. Epidural analgesia is provision of local anesthetic into the epidural space via an indwelling catheter [1]. Meperidine, also known as Demerol or Pethidine is a synthetic, phenylpiperidine class, opioid analgesic, administered intramuscularly.

Stage 1 labor is divided into latent and active phases. Currently in the United States, Stage 1, active phase labor may not begin until $6 \mathrm{~cm}$ cervical dilation [19]. Stage 2 labor is the period from $10 \mathrm{~cm}$ cervical dilation to the birth of the newborn. The newborn Appearance, Pulse, Grimace, Activity, and Respiration (APGAR) score at one and five minutes is an indication of neonatal wellbeing, with lower scores indicating a need for resuscitation of the newborn. Visual analog scales (VAS) use a position on a graduated or non-graduated continuous line, or meter, box, or graphic between two end-points to indicate agreement to a statement. Transcutaneous Electrical Nerve Stimulation (TENS) is the application of low-voltage electrical current at a topical pressure point or pain area to reduce pain.

\subsection{Acupuncture, Electro-Acupuncture, and Acupressure for Labor Pain Management}

Acupuncture's underlying mechanism of action has been previously described [20]. Labor may initiate meridian obstruction, which responds to acupuncture and acupressure [21]. Historically, three acupoints have been used for full-term LPM acupuncture and acupressure: Hegu (LI4), Sanyinjiao (SP6), and Zhiyin (B67) [21].

In addition to endogenous opioid stimulation, acupuncture and acupressure may stimulate oxytocin release contributing to biologically plausible reduced labor duration [22]. Acupuncture and acupressure can be used without contraindications in full-term Stage 1 labor [1]. Hegu (LI4), an acupoint on the dorsum of the hand, on the large intestine meridian, exerts physiologic effect by stimulating endorphin release [22]. For noninvasive EA, Sanyinjiao (SP6) is an acupoint of the calf, $5 \mathrm{~cm}$ above the medial malleolus [17]. For TCM acupuncture, Sanyinjiao (SP6) is at the intersection of the kidney, liver, and spleen meridians, which should lie on the tibial aspect of the leg, posterior to the medial tibial border and 3-bone cun superior to the medial malleolus [18]. Sanyinjiao (SP6) stimulates pituitary gland oxytocin release [22] [23]. Zhiyin (K1-B67) is an acupoint of the urinary bladder meridian at the intersection with the kidney meridian. Zhiyin (K1-B67) lies on the lateral small toe. Of note, the foregoing reviewed RCT do not explicitly mention trial use of Zhiyin (K1-B67).

A fourth acupoint series is frequently used for noninvasive EA: JaiaJin (EX-B2), which may also be denoted in the literature as JiaJin (T10-L3). JiaJin (EX-B2) are 34 acupoints lying about $1.7 \mathrm{~cm}$ bilateral to the posterior median line, between the tenth thoracic vertebrae and the third lumbar vertebrae [17]. Hence, the alternative designa- 
tion JiaJin (TL10-L3) [2]. For TCM acupuncture JiaJin (EX-B2) is $0.5 \mathrm{~cm}$ bone-cun bilateral to the lower border of each spinous process from the first thoracic vertebra to the fifth lumbar vertebra [18]. JiaJin (EX-B2 or TL10-L3) acts through spinal cord, dorsal horn, inhibitory or excitatory A fibers [23]. JiaJin (EX-B2 or TL10-L3) may be most effective for women experiencing back labor [23]. Zu san li (ST 36) is located 3 cun below the inferior edge of the patella, lateral to the patella ligament, one fingerbreadth from the anterior crest of the tibia. $\mathrm{Zu}$ san li (ST 36) activates serotonin modulation of large A-beta myelinated afferent fibers that suppress nociceptive unmyelinated C fibers [24].

Based on biologic plausibility, use of Sanyinjiao (SP6) would shorten labor by increasing uterine contractility, whereas use of Hegu (LI4) would decrease pain perception and decrease the need for analgesia [22]. Consistent with the aforementioned, it will be shown that Sanyinjiao is associated with quicker labor [21].

\section{Methods}

The PubMed database was searched on June 23, 2016 and November 10, 2016 using the terms "acupuncture treatment labor pain", with female, human subjects, age 19 to 44 years, English language, full text publication from 2012 onwards as parameters. Four English language RCTs were identified (see Figure 1). Academic One search on June 28, 2016 and November 10, 2016, with identical terms and similar parameters as above yielded a letter to the editor with a response pertaining to a RCT in the PubMed search. A Cumulative Index to Nursing and Allied Health Literature (CINAHL) search on June 28, 2016 and November 10, 2016, with identical terms and similar parameters found one review of systematic reviews (SR) from 2003 to 2011, and one RCT, as shown in Figure 1. Hand search found one meta-analysis and three RCT. The included RCT are summarized in Table 1 . The meta-analysis and review of SR are summarized in Table 2.

\section{Randomized Controlled Trials of Acupuncture, Electro-Acupuncture, and Acupressure for Labor Pain Management}

\subsection{Acupuncture versus Pethidine}

A registered, RCT of acupuncture and pethidine with 95 participants was performed at an Iranian hospital, from April to September, 2010 [25]. Randomization was by random number assignment. Eligible participants were at term with their first or second uncomplicated pregnancy, in labor, with a cervical dilation of 4 to $5 \mathrm{~cm}$, who had not used an analgesic in 4 hours. A $10 \mathrm{~cm}$ McGill pain ruler was used for pain scoring. Analgesics were not administered to the 27 control participants [25]. A single dose of Pethidine $50 \mathrm{mg}$ intramuscularly was received by 30 participants. The remaining 28 participants received bilateral MA at Hegu (LI4) and Zu san li (ST36) for 20 to 30 minutes. McGill pain ruler pain scores were recorded before and afterrandomization, in-between contractions, 30-minutes after intervention, and at $10 \mathrm{~cm}$ dilation [25]. 


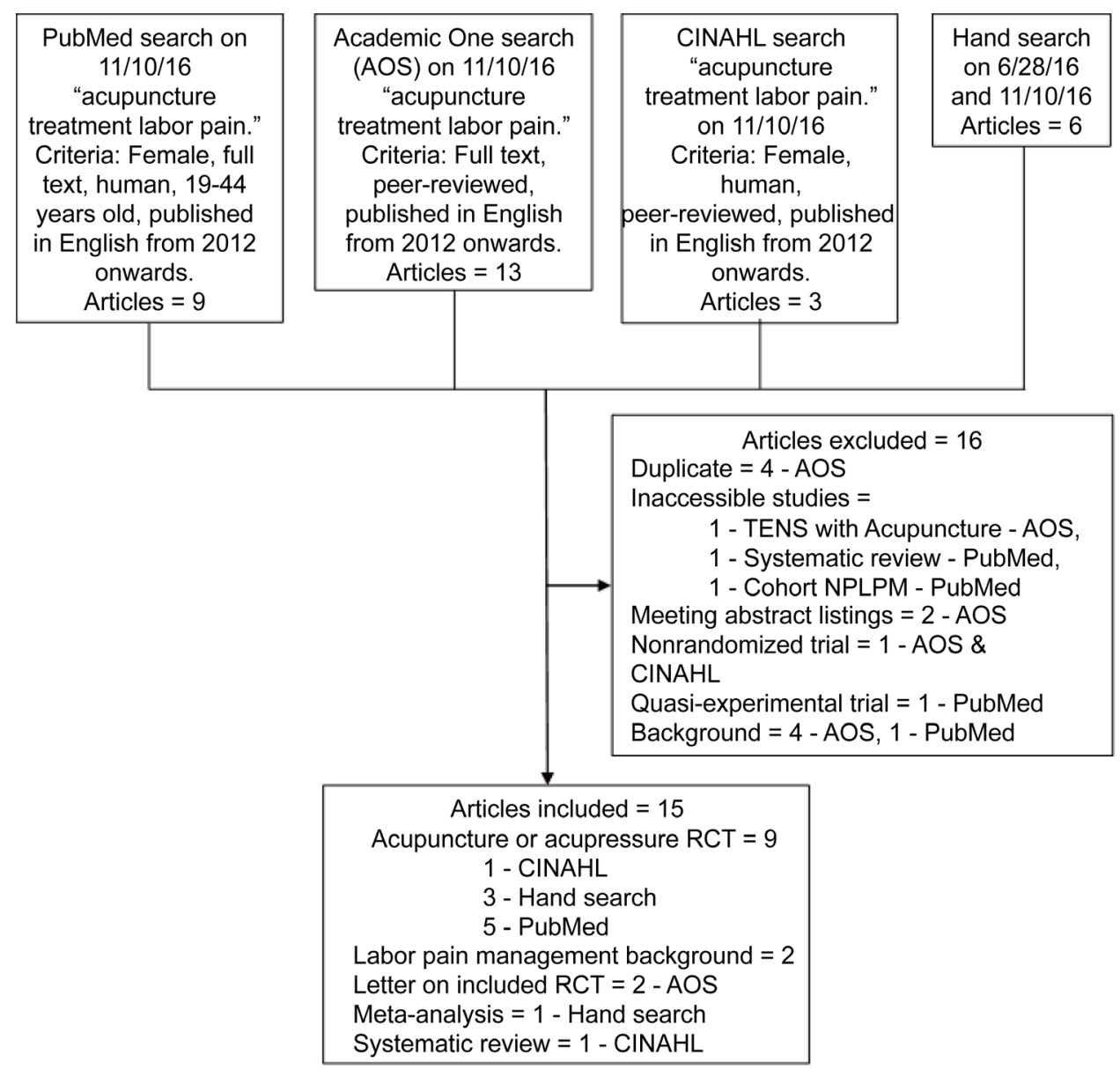

Figure 1. Article selection flowchart.

Statistically, parity, mean 1- and 5-minute APGAR scores, and pain scores before intervention and at $10 \mathrm{~cm}$ dilation were not significantly different across the groups. When compared to pethidine and no analgesic, bilateral MA most effectively reduced pain when measured 30 minutes after intervention, $p=0.0001$. In comparison to the control, bilateral MA and pethidine similarly reduced the mean length of active phase labor by 68 minutes, $p=0.0001$ [25]. This RCT by reference [25] is different from that by reference [26] in which acupuncture was repeated throughout labor, but similar to the RCT by references [2] and [21], in which participants received a single acupuncture or acupressure session. Limitations of the RCT by reference [25] include incomplete participant demographics, incomplete mention of membrane status and CD rates, and lack of mention of prior use of cervical ripening or inducing agents or post intervention use of labor augmenting agents.

\subsection{Acupuncture with Manual and Electrical Stimulation}

From November 2008 and October 2011, a registered RCT of 40 minutes of MA, or combined EA and MA, or standard care without acupuncture, for 303 nulliparas in spontaneous labor was conducted at two Swedish hospitals [26]. Study participation 
Table 1. Description of included original studies.

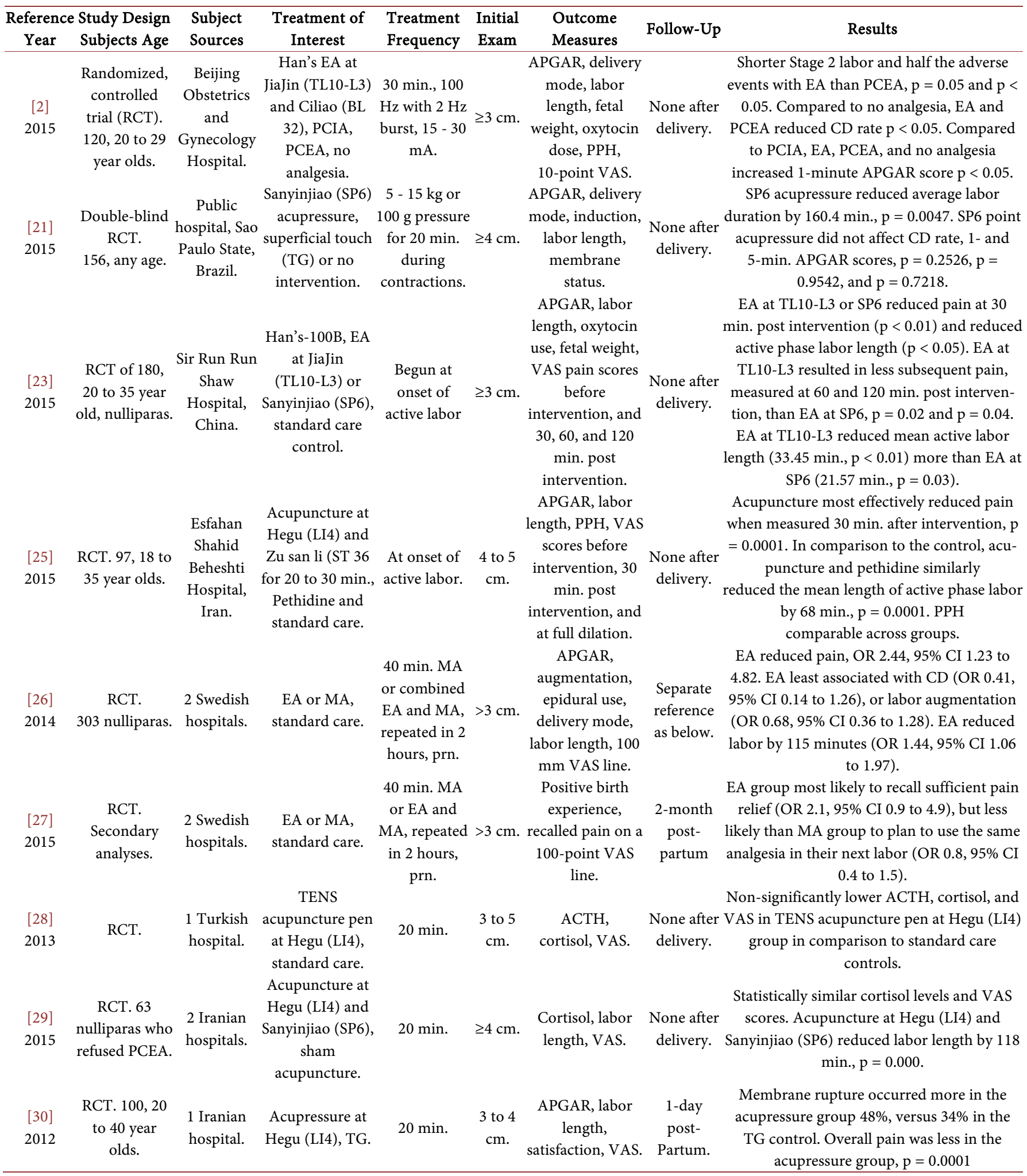

ACTH, adrenocorticotrophic hormone; APGAR, newborn Appearance, Pulse, Grimace, Activity, and Respiration score; CD, cesarean delivery; EA, electroacupuncture; MA, manual acupuncture; min., minutes; PCEA, patient-controlled epidural analgesia; PCIA, Patient-controlled intravenous analgesia; PPH, postpartum hemorrhage; prn, as needed; VAS, visual analog scale. 
Table 2. Description of included meta-analysis and critical narrative of systematic reviews.

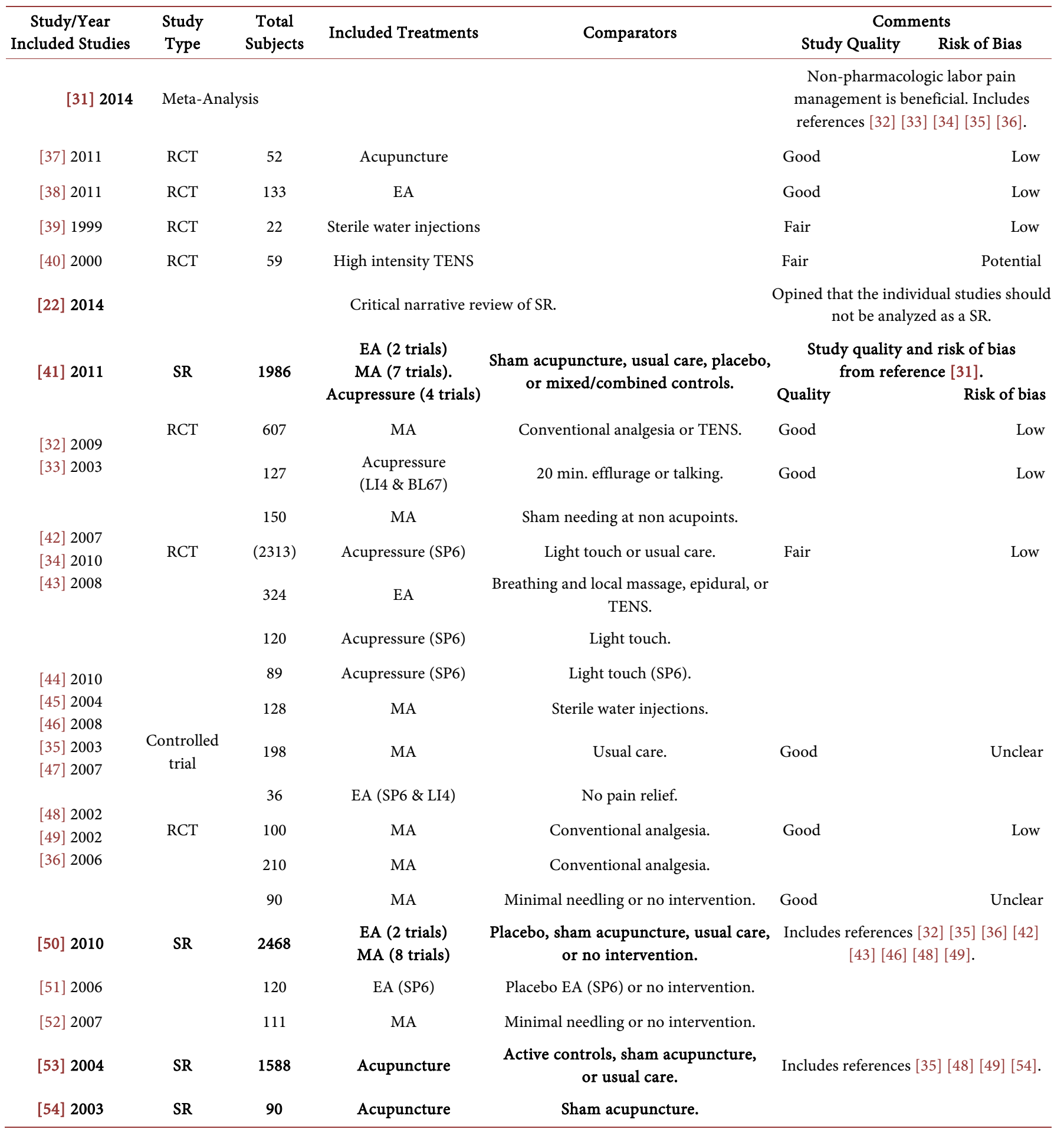

EA, electro-acupuncture, MA, manual acupuncture; RCT, randomized controlled trial; SR, systematic review; TENS, transcutaneous electrical nerve stimulation. Based on table 3 from reference [31], and table 1 and table 2 from reference [22].

was declined by 376 eligible patients. Women who had received oxytocin or analgesic other than acetaminophen prior to randomization were excluded. Random block com- 
puterized randomization assigned 99 participants to MA, 103 participants to combined EA and MA, and 101 participants to standard care. EA and MA involved 3 bilateral distal points, and 4-8 bilateral proximal points, with up to 21 needles applied [26]. MA comprised of manual stimulation to de Qi, then at 10 minute intervals until 40 minutes was reached. Combined EA and MA involved manual stimulation to de Qi, then $80 \mathrm{~Hz}$ stimulation at some needles, intensity adjusted by the recipient, with MA applied at all remaining needles. EA and MA protocols were repeated in 2 hours and subsequently upon recipient request. A $100 \mathrm{~mm}$ ungraded pain reporting line was used. Outcomes from a 2-months postpartum evaluation were presented separately [27].

Standard care recipients were older and more educated than MA recipients. Otherwise the groups were identical. Compared to MA recipients, combined EA and MA recipients were more likely to rate acupuncture as effective for reducing pain (Odds ratio (OR) 2.44 , 95\% confidence interval (CI) 1.23 to 4.82), and for relaxation (OR 1.72, 95\% CI 0.84 to 3.52) [26]. Combined EA and MA recipients were least likely to use epidural analgesia (OR 0.35 , 95\% CI 0.19 to 0.67 ), have a CD (OR 0.41, 95\% CI 0.14 to 1.26 ), or require labor augmentation (OR $0.68,95 \%$ CI 0.36 to 1.28 ), and had the shortest labor by 115 minutes (OR 1.44, 95\% CI 1.06 to 1.97). However, combined EA and MA recipients had the highest mean pain score at the furthest time from baseline, possibly associated with the MA and standard care groups receiving more epidurals, and the MA and standard care groups receiving epidurals at an earlier time from baseline than the combined EA and MA group [26]. In comparison to standard care, the least newborn referrals to the neonatal clinic were from the MA group (OR 0.91, 95\% CI 0.19 to 4.31), whereas the most newborn referrals to the neonatal clinic were from the EA group (OR $2.8295 \%$ CI 0.82 to 9.68$)$.

In the two-month postpartum follow-up study, the combined EA and MA group was most likely to recall sufficient pain relief (OR 2.1, 95\% CI 0.9 to 4.9), confirming that the combined EA and MA group's lower epidural use reflected adequately treated labor pain [27]. Negative birth experiences were identical across groups. However, the combined EA and MA group was less likely than the MA group to plan to use the same analgesia in their next labor (OR 0.8, 95\% CI 0.4 to 1.5) [27]. These results are summarized in Table 3. Intent to treat analysis is a strength of both studies [26] [27]. The initial study has several limitations [26]. Use of multiple acupoints and varying number of acupuncture needles per recipient impairs study replication. While Sanyinjiao (SP6) was included, but only used in $33.7 \%$ of MA and $36.8 \%$ of EA recipients, JiaJin (EX B2) and Ciliao (BL32) were not included, impairing comparison with all included studies [2] [21]. Electro-acupuncture and/or MA application on recipient demand obscures a reproducible dosing regime.

\subsection{Noninvasive Electro-Acupuncture at Hegu (LI4)}

A pilot RCT of 20 minutes EA at Hegu (LI4) compared to standard intrapartum care was performed over six months in 2010, at a single Turkish hospital [28]. Low risk term 
Table 3. Combined electro-acupuncture (EA) with manual acupuncture (MA), versus MA only, and standard care (SC) outcomes.

\begin{tabular}{|c|c|c|c|c|}
\hline \multirow[b]{2}{*}{ Outcome } & \multicolumn{2}{|c|}{ EA with MA versus MA only } & \multicolumn{2}{|c|}{ EA versus SC } \\
\hline & Odds Ratio & $\begin{array}{c}\text { 95\% Confidence } \\
\text { Interval }\end{array}$ & Odds Ratio & $\begin{array}{l}\text { 95\% Confidence } \\
\text { Interval }\end{array}$ \\
\hline Pain reduction effectiveness & 2.44 & 1.23 to 4.82 & & \\
\hline Choose method again & 1.14 & 0.55 to 2.36 & & \\
\hline Sufficient analgesia & 1.28 & 0.58 to 2.84 & 1.68 & 0.58 to 2.84 \\
\hline Epidural analgesia used & 0.57 & 0.31 to 1.06 & & \\
\hline Cesarean delivery incidence & 0.65 & 0.20 to 2.14 & 0.41 & 0.14 to 1.26 \\
\hline Labor augmentation needed & 0.68 & 0.36 to 1.28 & 0.81 & 0.43 to 1.51 \\
\hline Shortest labor by 115 minute & 1.41 & 1.03 to 1.91 & 1.44 & 1.06 to 1.97 \\
\hline Neonatal clinic referrals & 3.11 & 0.81 to 11.98 & 2.82 & 0.82 to 9.68 \\
\hline \multicolumn{5}{|l|}{ 2-month Follow-up Outcome } \\
\hline Recall sufficient pain relief & 1.7 & 0.7 to 4.0 & 2.1 & 0.9 to 4.9 \\
\hline Likelihood to repeat analgesia & 0.8 & 0.4 to 1.5 & & \\
\hline
\end{tabular}

Data extracted from reference [26] table 4 and table 5, and reference [27] table 2.

pregnancy with presentation to labor and delivery at 3 to $5 \mathrm{~cm}$ cervical dilation was required for study inclusion. Randomization was by choice of shuffled, opaque, sealed envelopes containing RCT intervention assignment. While 50 participants were randomized to each group, analysis is based on 39 participants per group [28]. A VAS from 0 to 10 was used for pain measurement. The noninvasive XFT-320 TENS-acupuncture pen was used to perform EA. Dense-dispersed waveform frequency was 5 to $10 \mathrm{~Hz}$, with a 2.5 millisecond burst, and maximum intensity of $0.6 \mathrm{~mA}$.

The EA group had significantly lower pre-intervention pulse at 83.7 beats per minute, $\mathrm{p}=0.007$ [28]. Pre-intervention morning adrenocorticotrophic hormone (ACTH) and cortisol levels were statistically not different, but were lower in the EA group. While between group pre-intervention and serial post-intervention pain scores were not statistically different, there was a trend towards increasingly less pain in the EA group.

This pilot RCT is limited by failure to perform intent to treat analysis and postrandomization exclusion of participants receiving cesarean delivery or oxytocin augmentation, experiencing precipitous labor, and who refused to provide blood samples, or whose blood samples did not yield useable results [28]. Larger sample size may be necessary to verify real differences between EA and standard intrapartum care. Randomization via intervention assignment containing envelopes is criticized the assignment may not be concealed and is subject to conscious and subconscious sabotage. Concealed assignment is preferable [55].

\subsection{Noninvasive Electro-Acupuncture at JiaJin (TL10-L3) and Ciliao (BL 32)}

A RCT run from August 2010 through November 2013, compared 30 minutes $100 \mathrm{~Hz}$ 
direct current, 15 to $30 \mathrm{~mA}$ pulse stimulation of JiaJin (TL10-L3) and Ciliao (BL 32) by Han's acupoint nerve stimulator, to patient controlled intravenous analgesia (PCIA), patient-controlled epidural analgesia (PCEA), and a non-analgesia control group [2]. A tramadol and ondansetron combination formed the PCIA. Ropivacaine and Sufentanil comprised the PCEA. Random number table randomization assigned 30 participants to each of four groups. Participants were excluded for analgesic allergies, poor prior obstetric outcomes, current high risk gestations, chronic analgesic, anxiolytic, or sedative use, body mass index (BMI) outside the normal range, and inability to agree to natural labor [2].

Demographics and neonatal weights for all four groups were identical. At the cost of the longest second stage of labor, pruritus, neonatal asphyxia, and urinary retention, PCEA achieved the greatest analgesic effect, $\mathrm{p}=0.05$. However, at all measured points (30- and 60-minutes post intervention, 7 to $8 \mathrm{~cm}$ and $10 \mathrm{~cm}$ dilation), Han's acupoint nerve stimulator recipients had lower 10-point VAS points than did no analgesia control group participants (26, 32, 30, and 30, respectively), $\mathrm{p}<0.05$ [2]. Moreover, Han's acupoint nerve stimulator recipients had a 17.9-minute shorter Stage 2 labor than did PCEA recipients, $\mathrm{p}=0.05$ [2]. Any form of analgesia reduced the need for cesarean delivery in comparison to no analgesia, $\mathrm{p}<0.05$, whereas PCIA reduced the need for oxytocin augmentation, $\mathrm{p}<0.05$, but achieved the lowest 1 minute APGAR score, $\mathrm{p}<$ 0.05 . The most adverse effects were experienced by the PCIA group, with PCEA recipients experiencing half as many adverse effects than PCIA recipients, $\mathrm{p}<0.05$. Control and Han's acupoint nerve stimulator recipients experienced half as many adverse effects than the PCEA group, $\mathrm{p}<0.05$ [2].

Reference [2] is limited by the classification of active phase, Stage 1 labor starting at 3 $\mathrm{cm}$, when 5 to $6 \mathrm{~cm}$ may now be considered active phase Stage 1 labor [19]. Reference [2] is further limited by study restriction to patients naïve to acupuncture. It is possible that women who have had previous positive acupuncture outcomes may wish to use acupuncture for labor analgesia. Such women could reasonably want to know what outcomes for acupuncture exposed women are with acupuncture for labor analgesia.

\subsection{Acupuncture at Hegu (LI4) and Sanyinjiao (SP6)}

From October 2011 through October 2012, 71 low-risk nulliparas requesting natural labor at 37 or greater weeks estimated gestational age at two hospitals in Iran, underwent simple 1:1 randomization by hospital admission code to MA for 20 minutes at Hegu (LI4) and Sanyinjiao (SP6) or standard care [29]. At 4 or more $\mathrm{cm}$ dilation 35 participants received the intervention and 36 control participants received sham acupuncture, but data was analyzed for 32 and 31 participants, respectively. Participants receiving $\mathrm{CD}$ or who discontinued participation for other reasons were excluded from all analysis. Serial pain measurements were taken using a 10-point VAS. Serum cortisol was measured pre- and 1-hour post-intervention, with both samples taken between 6 $\mathrm{pm}$ and $10 \mathrm{pm}$ [29]. The analyzed groups were demographically and physiologically identical at baseline. Post-intervention serum cortisol and pain levels remained similar. First stage labor duration was not distinctly tabulated, but mean total labor length was 
118 minutes less in the intervention group, with a mean of 162 minutes, $95 \%$ CI 146 to 177 minutes, versus the control group mean of 280 minutes, 95\% CI 257 to $304 \mathrm{mi}-$ nutes, $\mathrm{p}=0.000$ [29]. Selection bias, small group sizes, lack of intent to treat analysis, and possible typographical errors in the publication are study limitations. Therefore, this RCT is not perceived as of sufficient rigor to base future treatment recommendations.

\subsection{Noninvasive Electro-Acupuncture at JiaJin (TL10-L3) or Sanyinjiao (SP6)}

From October 2012 to September 2013, a RCT of bilateral EA at JiaJin (TL10-L3) or Sanyinjiao (SP6) versus standard intrapartum care, with 60 nulliparas at $3 \mathrm{~cm}$ or greater cervical dilation per group, was performed at a single Chinese hospital [23]. Prior EA use, high risk pregnancy, and pacemaker use were pre-randomization exclusion criteria. Post-randomization, inevitable cesarean delivery or precipitous labor led to participant exclusion. Pain was measured by a $10 \mathrm{~cm}$ VAS. Han's EA device with electrode pads, $100 \mathrm{~Hz}$, burst frequency $2 \mathrm{~Hz}$, at a minimum $15 \mathrm{~mA}$ current intensity was used [23].

Demographically, the three groups were homogenous. Compared to control, EA at either Jiajin (TL10-L3) or Sanyinjiao (SP6) significantly reduced pain as measured by VAS at 30-minutes post intervention $(\mathrm{p}<0.01)$ and reduced Stage 1 active phase labor length ( $\mathrm{p}<0.05)$. However, EA at Jiajin (TL10-L3) resulted in less subsequent pain, measured at 60 and 120-minutes post intervention, than did EA at Sanyinjiao (SP6), p $=0.02$ and $\mathrm{p}=0.04$, respectively. In comparison to standard antepartum care, EA at Jiajin (TL10-L3) also resulted in greater reduction in mean Stage 1 active labor length (33.45 minutes, $\mathrm{p}<0.01$ ) than did EA at Sanyinjiao (SP6; 21.57 minutes, $\mathrm{p}=0.03$ ) [23]. Across groups there was not statistical difference in duration of stage 2 and 3 labor, augmentation use, neo-natal weight, or APGAR scores.

Post-randomization exclusion criteria based analysis instead of intent to treat analysis is a study limitation [23]. Small group sizes were used [23]. Use of a single acupoint in each intervention group is a treatment limitation [23], however, single acupoints per group permits differentiation of an acupoint's efficacy. JiaJin (EX-B2 or TL10-L3) may be the go to acupoint for patients having back labor. A subsequent RCT may evaluate synergism of JiaJin (EX-B2), Ciliao (BL 32), and Sanyinjiao (SP6).

\subsection{Acupuncture at Hegu (LI4)}

From September to December 2006, 100 women at 37 or greater weeks estimated gestational age at a public teaching hospital in Iran underwent stratified randomization in blocks of 2, to bilateral acupressure at Hegu(LI4) or bilateral light touch at Hegu(LI4), 50 participants per group [30]. Substance abuse, three previous deliveries, prior CD, complicated obstetric history, and receipt of analgesic or augmentation in the current labor before study participation at 3 to $4 \mathrm{~cm}$ dilation were the main exclusion criteria. Five 3 to $5 \mathrm{~kg}$ pressures per minute for 20 minutes formed the intervention. Numerous 10-point VAS measurements were taken [30]. 
Demographically, the groups were identical. However, spontaneous membrane rupture occurred proportionally more in the acupressure group ( $48 \%$ versus $34 \%, p>0.05$, exact value not provided), consistent with 38 minutes shorter duration of first stage labor in the acupressure group, $\mathrm{p}<0.001$ [30]. Therefore, shortened labor duration in this RCT may not be attributable to the acupressure intervention, which is a study limitation. The acupressure group also had an 8 minute shorter second stage labor, $\mathrm{p}=$ 0.038. Newborn weight, 1- and 5-minute APGAR scores were not statistically different. From the intervention through 120 minutes post-intervention, the acupressure group had significantly lower 10 -point VAS scores, $\mathrm{p}<0.001$. Overall, and one day postpartum the acupressure group had lower labor pain perception, $\mathrm{p}=0.0001$ [30].

\subsection{Acupuncture at Sanyinjiao (SP6)}

From January to August 2013, 156 women at 37 weeks estimated gestational age at a public teaching hospital in Sao Paulo state, Brazil, participated in a double-blind RCT of 20 minutes bilateral Sanyinjiao SP6 point acupressure and bilateral SP6 acupoint touch placebo (TG) during contractions, with an un-blinded control group [21]. Randomization of 52 participants per group was by random number list. There were neither age nor parity exclusions. Women at $4 \mathrm{~cm}$ or more dilation with 2 to 3 contractions in 10 minutes without contraindication for bilateral SP6 point acupressure were included. High risk pregnancies, including women with two or more previous CD were excluded [21]. Brisk and rapid decompression medium-intensity (5 to $15 \mathrm{~kg}$ ) acupressure was performed with the index finger. Very-low-intensity pressure $(100 \mathrm{~g})$ was performed for the TG. This study was designed to assess labor duration and cesarean section rate. This study was included as the longer a patient labors, the longer she is in pain.

Compared to control, bilateral Sanyinjiao SP6 acupoint acupressure reduced average labor duration by 160.4 minutes, $\mathrm{p}=0.0047$. Bilateral Sanyinjiao SP6 point acupressure did not affect CD rate, 1 - and 5-minute APGAR scores, $\mathrm{p}=0.2526, \mathrm{p}=0.9542$, and $\mathrm{p}=$ 0.7218 respectively. This study is limited by control subjects' lower parity, $\mathrm{p}=0.0232$, the TG subjects' being more likely to have intact amniotic membranes, $\mathrm{p}=0.0416$, and the lack of intention-to-treat analysis [21]. Therefore, it reduced average labor duration in the intervention group cannot be attributed to the intervention, bilateral Sanyinjiao SP6 acupoint acupressure.

\section{Meta-Analysis and Systematic Reviews of Acupuncture, Electro-Acupuncture, and Acupressure for Labor Pain Management}

\subsection{Meta-Analysis of Non-Pharmacologic Labor Pain Management}

Five acupuncture, one EA, and two acupressure RCT, totaling 3590 participants from east Asia, western-Europe, Scandinavia, and the Middle East were included in a multimodal intervention meta-analysis of NPLPM [31]. Intervention implementation quality based on the Bonapace and Marchand classifications and standardized with possible 
ratings "Poor", "Fair", or "Good", was given as good for seven RCT and fair for one RCT. Due to painful stimulation use, the Bonapace and Marchand Classification categorizes acupuncture, acupressure in the diffuse noxious inhibitory control theoretical model (DNIC). Based on the Cochrane and Effective Practice and Organization of Care Risk of Bias Tool, risk of bias was listed as low for six RCT and unclear for three RCT: None of the included RCT received a potential risk of bias rating [31].

When compared to DNIC category treatments, usual intrapartum care increased epidural use (OR 1.62 (95\% CI 1.18 to 2.21 ), increased labor pain (mean difference (MD) on a 0 to 100 VAS score of $10.3,95 \%$ CI 4.7 to 15.9), and decreased maternal satisfaction with the childbirth experience (MD $-8.8,95 \% \mathrm{CI}-17.06$ to -0.54 ). However, this meta-analysis is limited by the inclusion of a fair quality, potentially biased, RCT of high intensity TENS, and a fair quality, low risk of bias, RCT of sterile water injections in the analysis of acupuncture, acupressure, and electro-acupuncture RCT, in the only presented DNIC category analyses [31]. When noninvasive EA is performed with TENS, smaller electrodes positioned at acupoints are used, whereas for TENS larger electrodes are used, which are not necessarily placed at acupoints [56]. Similarly, sterile water placement may or may not occur at acupoints [56]. The resultant statistical analyses showing increased epidural use, increased labor pain, and decreased maternal satisfaction with childbirth, in usual care participants when compared to NPLPM participants, cannot be directly compared to an OR from a meta-analysis restricted to acupuncture, acupressure, and electro-acupuncture. This meta-analysis may have achieved greater significance if the DNIC category analysis had been performed on a sub-group and a group level [31].

\subsection{Critical Narrative Review of Systematic Reviews}

Reference [22] reviewed four systematic reviews from 2003 to 2011, incorporating 16 original trials, and 5,023 unique participants. Six of the eight RCT reviewed in reference [31] are included [22]. The electro-acupuncture RCT and the smallest acupuncture RCT reviewed in reference [31] were not included [22].

The critical narrative is limited by inconsistencies between the text and the tables, especially, participant numbers [22]. Heterogeneity in the outcomes analyses for each of the four incorporated systematic reviews, means that despite duplication of included original trials, the resultant outcomes data may not be directly comparable. However, use of the acupoint Sanyinjiao (SP6) was associated with increased uterine contractility, consistent with physiologic mechanism of action by stimulating pituitary gland oxytocin release [22]. Hegu (LI4) acupoint use was also associated with lower pain intensity scores, consistent with physiologic mechanism of action by stimulating endorphin release [22].

\section{Efficacy and Adverse Effects of Epidural Regional Analgesia for Labor Pain Treatment}

Meta-analysis shows that operative vaginal deliveries are increased by epidural analge- 
sia, relative risk $(\mathrm{RR})=1.42,95 \%$ CI 1.28 to 1.57 [1]. Second stage epidural discontinuation impairs labor analgesia without removing the increased operative vaginal delivery risk [1]. However, for near-term and term induced nulliparas, epidural analgesia does not increase labor duration or $\mathrm{CD}$ rate [1]. Unlike acupuncture, EA, and acupressure that are recommended for first stage of labor use, epidural analgesia can be used in both the first and second stages of labor [1]. Hypovolemia, local anesthetic allergy, injection site skin infection, and coagulopathy are contraindications to epidural analgesia [1]. Epidural analgesia may cause hypotension, lumbago, fever, post dural puncture spinal headache, nerve injury, and paralysis [1]. Reference [2] showed that while theoretically PCEA has the potential for greater adverse events than PCIA, in practice PCIA can have a worse adverse event profile than PCEA.

\section{Discussion}

Single performance of bilateral MA at Hegu (LI4) and Zu san li (ST 36) for 20 to 30 minutes can significantly reduce duration of labor from $4 \mathrm{~cm}$ dilation by 68 minutes [25]. Single performance of bilateral acupressure at Hegu (LI4) for 20 minutes can significantly reduce overall 10-point VAS pain measurement from 3 to $4 \mathrm{~cm}$ dilation through second stage labor, and 24-hour post-partum perception thereof, $\mathrm{p}=0.001$ [30]. Serial application of bilateral EA combined with MA at multiple points can reduce labor by 115 minutes (OR 1.44, 95\% CI 1.06 to 1.97) [26]. A single 30-minute application of Han's EA at JiaJin (TL10-L3) and Ciliao (BL 32) reduces stage 2 labor by 17.9 minutes in comparison to labor duration with PCEA, $\mathrm{p}=<0.05$ [2].

Future research should display study design consistency, facilitating comparison across RCTs, which is currently problematic [22]. Instead of multiple pain scales (an ungraded $100 \mathrm{~mm}$ pain line, the McGill $10 \mathrm{~cm}$ pain ruler, a 10-point VAS, and 0 to 100 VAS), a single pain scale should be used [2] [25] [26] [27]. Membrane status was included in four RCT [2] [21] [26] [30], but not included in five RCT [23] [25] [27] [28] [29]. Membrane status should be uniformly reported across all future RCT. The RCT described above used different set points for onset of active phase labor, which makes comparison of duration of active phase labor difficult. For instance, references [2] and [23] used $\geq 3 \mathrm{~cm}$ dilation, and reference [21] used $\geq 4$ or more $\mathrm{cm}$ dilation, whereas reference [25] used 4 to $5 \mathrm{~cm}$ dilation. Although this inconsistency is consistent with the literature, for research purposes a single set point for the onset of active phase Stage 1 labor is needed [19] [57]. While references [2] and [23], used Han's EA device, reference [28] used the XFT-320 TENS unit at different settings to the Han's EA device. Use of different EA devices, and different settings on the EA devices, limits direct comparison across studies.

Future RCT could also improve upon the literature. Group and sub-group size could be increased to a minimum of 60 participants [23]. Given the current global trend towards obesity, RCT should include overweight and obese patients. Computer-generated randomization with allocation concealment is preferable to the use of intervention assignment containing envelopes [55]. Although seemingly ideologically contrary to 
TCM, to facilitate reproducible RCT, reproducible dosing regimes with a limited number of acupoints and a fixed dosing pattern are needed. Post-randomization participant exclusion reduces the level of evidence. Intent to treat analysis is preferable.

Several areas of future research are clear. EA device and setting equivalence studies would limit the need to use identical EA devices in future studies, providing investigator and facility flexibility. Synergism of EA at Jiajin (TL10-L3), Ciliao (BL 32), and Sanyinjiao (SP6) should be evaluated by RCT. Additional RCT evaluating stand-alone EA efficacy of JiaJin (TL10-L3), Ciliao (BL 32), and Sanyinjiao (SP6) are needed to support or refute references [2] and [23]. Additional RCT of acupressure at Sanyinjiao (SP6) to support or refute reference [21], and add analgesia data to complement labor duration data are needed. Each EA protocol should be trialed against a MA protocol to ascertain if EA is more advantageous than MA.

Future studies on acupuncture or acupressure for labor pain management may consider addition of oxytocin level measurement into study protocols to ascertain if in fact acupuncture and acupressure increase laboring women's oxytocin levels. Future RCT participants receiving epidural analgesia and/or delivering via cesarean should be included in trial protocols and intent to treat analysis as comparative efficacy of NPLPM modalities to regional analgesia, and the association if any, between NPLPM and CD rates should be thoroughly assessed. As none of the included RCT expressly included participants with current or remote substance abuse, future RCT should consider including participants with a history of substance abuse to evaluate comparative efficacy of acupuncture and acupressure for all patient populations. Future studies could consider combining acupuncture or acupressure with regional analgesia to evaluate if the labor shortening effects of acupuncture and acupressure balance the labor prolonging effects of regional analgesia. More studies on acceptability of the available array of NPLPM modalities could better describe interest, while also informing health care providers and facilities as to what to offer pregnant women.

\section{Conclusions}

Electro-acupuncture at Ciliao (BL 32) and Jiajin (TL10-L3), or JiaJin (TL10-L3) and/or Sanyinjiao (SP6), is a reasonable option for women who prefer not to use PCEA or PCIA, but do not wish for a completely natural labor process. Electro-acupuncture at these acupoints can reduce Stage 1 active labor length $(\mathrm{p}<0.05)$ and some post-intervention pain scores ( $\mathrm{p}<0.01$ ) [2] [23]. Bilateral MA at Hegu (LI4) and Zu san li (ST 36) may also effectively reduce Stage 1 active labor length and pain scores in comparison to intramuscular Pethidine [25]. As only one RCT with 2-month follow-up compared the combination of EA and MA versus MA [26] [27], and none compared EA to MA, there is insufficient data to determine which intervention is most advantageous.

Due to demographic and physiologic disparities between the intervention and control groups, bilateral acupressure at Sanyinjiao (SP6) cannot be recommended without corroborating studies [21]. Due to physiologic disparities between the intervention and control groups, bilateral acupressure at Hegu (LI4) should not be recommended with- 
out corroborating studies [30]. JiaJin (TL10-L3) may be the go to acupoint for patients having back labor [23]. Noninvasive EA at Hegu (LI4) needs further investigation with more than 40 participants per group. Consistent with biologic plausibility, Sanyinjiao (SP6) was associated with quicker labor, but association between Sanyinjiao and reduced CD rate was not corroborated [21]. Due to increased uterine contractility, Sanyinjiao (SP6) may not be associated with as much pain reduction as Hegu (LI4) or JiaJin (TL10-L3). Consistent with biologic plausibility, Hegu (LI4) would decrease pain perception and decrease the need for analgesia [22].

In 2011, the Cochrane Review found that acupuncture and acupressure may have a role in reducing labor pain [22] [41]. Current evidence indicates that EA should have a role in NPLP, and that acupressure may have a role in NPLPM. Future RCTs could strengthen the argument for increased use of EA and acupressure in NPLPM. Public awareness of and access to NPLPM should be increased.

\section{Acknowledgements}

This paper is based on a presentation at the American Public Health Association 2016 Annual Meeting and Expo, Denver, October 29-November 2, 2016.

\section{References}

[1] Schrock, S.D. and Harraway-Smith, C. (2012) Labor Analgesia. American Family Physician, 85, 448-454.

[2] Ye, L., Xu, M., Che, X., He, J., Guo, D., Zhao, G., et al. (2015) Effect of Direct Current Pulse Stimulating Acupoints of JiaJi (T10-L3) and Ciliao (BL 32) with Han's Acupoint Nerve Stimulator on Labour Pain in Women: A Randomized Controlled Clinical Study. Journal of Traditional Chinese Medicine, 35, 620-625. https://doi.org/10.1016/S0254-6272(15)30149-7

[3] Saia, K., Schiff, D., Wachman, E.M., Mehta, P., Vilkins, A., Sia, M., et al. (2016) Caring for Pregnant Women with Opioid Use Disorder in the USA: Expanding and Improving Treatment. Current Obstetrics and Gynecology Reports, 5, 257-263.

https://doi.org/10.1007/s13669-016-0168-9

[4] Jones, C.M., Logan, J., Gladden, R.M. and Bohm, M.K. (2015) Vital Signs: Demographic and Substance Use Trends among Heroin Users-United States, 2002-2013. Morbidity and Mortality Weekly Report, 64, 719-725. http://www.cdc.gov/mmwr/preview/mmwrhtml/mm6426a3.htm

[5] Unger, A., Metz, V. and Fischer, G. (2012) Opioid Dependent and Pregnant: What Are the Best Options for Mothers and Neonates? Obstetrics and Gynecology International, 2012, Article ID: 195954. https://doi.org/10.1155/2012/195954

[6] Krans, E.E. and Patrick, S.W. (2016) Opioid Use Disorder in Pregnancy. Health Policy and Practice in the Midst of an Epidemic. Obstetrics \& Gynecology, 128, 4-10. https://doi.org/10.1097/AOG.0000000000001446

[7] Tolia, V.N., Patrick, S.W., Bennett, M.M., Murthy, K., Sousa, J., Smith, B., et al. (2015) Increasing Incidence of the Neonatal Abstinence Syndrome in US Neonatal ICUs. The New England Journal of Medicine, 372, 2118-2126. https://doi.org/10.1056/NEJMsa1500439

[8] Reddy, U.M. (2016) Screening, Prevention, and Treatment of Opioid Use Disorder during Pregnancy. Expectant Mothers are Depending on You! Obstetrics \& Gynecology, 128, 1-3. https://doi.org/10.1097/aog.0000000000001505 
[9] Sedgh, G., Singh, S. and Hussain, R. (2014) Intended and Unintended Pregnancies Worldwide in 2012 and Recent Trends. Studies in Family Planning, 45, 301-314.

https://doi.org/10.1111/j.1728-4465.2014.00393.x

[10] The American College of Obstetricians and Gynecologists Committee on Health Care for the Underserved Women and the American Society of Addiction Medicine (2012) Opioid Abuse, Dependence, and Addiction in Pregnancy. Obstetrics \& Gynecology, 119, 1070 1076. https://doi.org/10.1097/AOG.0b013e318256496e

[11] Taylor, D.R. (2015) Managing Patients with Chronic Pain and Opioid Addiction. Springer, Basel. https://doi.org/10.1007/978-3-319-08111-3

[12] Anarado, A., Ali, E., Nwonu, E., Chinweuba, A. and Ogbolu, Y. (2015) Knowledge and Willingness of Prenatal Women in Enugu Southeastern Nigeria to Use in Labour Non-Pharmacological Pain Reliefs. African Health Sciences, 15, 568-575.

https://doi.org/10.4314/ahs.v15i2.32

[13] Brown, S.T., Douglas, C. and Flood, L.P. (2001) Women's Evaluation of Intrapartum NonPharmacological Pain Relief Methods Used during Labor. The Journal of Perinatal Education, 10, 1-8. https://doi.org/10.1624/105812401X88273

[14] Foden-Vencil, K. (2015) To Curb Pain without Opioids, Oregon Looks to Alternative treatments.

http://www.npr.org/sections/health-shots/2015/09/22/436905063/to-curb-pain-without-opi oids-oregon-looks-to-alternative-treatments

[15] Meier, B. and Goodnough, A. (2016) New Ways to Treat Pain Meet Resistance. The New York Times.

http://www.nytimes.com/2016/06/23/business/new-ways-to-treat-pain-without-opioids-me et-resistance.html?_r=0

[16] Perlin, C. (2015) The Truth about Chronic Pain Treatments. Morning Light Books, New York.

[17] Dong, C. (2015) Response to: "Electro-Acupuncture Effectiveness on Labor Pain Management". Archives of Gynecology and Obstetrics, 292, 237. https://doi.org/10.1007/s00404-015-3735-9

[18] Sha, T., Gao, L., Zheng, J. and Meng, Z. (2015) Electro-Acupuncture Effectiveness on Labor Pain Management. Archives of Gynecology \& Obstetrics, 292, 235-236. https://doi.org/10.1007/s00404-015-3733-y

[19] Zhang, J., Landy, H.J., Branch, D.W., Burkman, R., Haberman, S., Gregory, K.D., et al. (2010) Contemporary Patterns of Spontaneous Labor with Normal Neonatal Outcomes. Obstetrics and Gynecology, 116, 1281-1287. https://doi.org/10.1097/AOG.0b013e3181fdef6e

[20] Nwanodi, O.B. and Tidman, M.M. (2014) Vulvodynia Treated with Acupuncture or Electromyographic Biofeedback. Chinese Medicine, 5, 61-70. https://doi.org/10.4236/cm.2014.52007

[21] Mafetoni, R.R. and Shimo, A.K.K. (2015) Effects of Acupressure on Progress of Labor and Cesarean Section Rate: Randomized Clinical Trial. Revista de Saúde Pública, 49, 9. https://doi.org/10.1590/S0034-8910.2015049005407

[22] Levett, K.M., Smith, C.A., Dahlen, H.G. and Bensoussan, A. (2014) Acupuncture and Acupressure for Pain Management in Labor and Birth: A Critical Narrative Review of Current Systematic Review Evidence. Complementary Therapies in Medicine, 22, 523-540. https://doi.org/10.1016/j.ctim.2014.03.011

[23] Dong, C., Hu, L., Liang, F. and Zhang, S. (2015) Effects of Electro-Acupuncture on Labor 
Pain Management. Archives of Gynecology and Obstetrics, 291, 531-536.

https://doi.org/10.1007/s00404-014-3427-x

[24] Chang, F.C., Tsai, H.Y., Yu, M.C., Yi, P.L. and Lin, J.G. (2004) The Central Serotonergic System Mediates the Analgesic Effect of Electroacupuncture on Zusanli (ST36) Acupoints. Journal of Biomedical Science, 11, 179-185. https://doi.org/10.1159/000076030

[25] Allameh, Z., Tehrani, H.G. and Ghasemi, M. (2015) Comparing the Impact of Acupuncture and Pethidine on Reducing Labor Pain. Advanced Biomedical Research, 4, 46. https://doi.org/10.4103/2277-9175.151302

[26] Vixner, L., Schytt, E., Sterner-Victorin, E., Waldenström, U., Pettersson, H. and Mårtensson, L.B. (2014) Acupuncture with Manual and Electrical Stimulation for Labour Pain: A Longitudinal Randomised Controlled Trial. BMC Complementary \& Alternative Medicine, 14, 187. https://doi.org/10.1186/1472-6882-14-187

[27] Vixner, L., Mårtensson, L.B. and Schytt, E. (2015) Acupuncture with Manual and Electrical Stimulation for Labour Pain: A Two Month Follow up of Recollection of Pain and Birth Experience. BMC Complementary \& Alternative Medicine, 15, 180. https://doi.org/10.1186/s12906-015-0708-2

[28] Mucuk, S., Baser, M. and Ozkan, T. (2013) Effects of Noninvasive Electroacupuncture on Labor Pain, Adrenocorticotropic Hormone, and Cortisol. Alternative Therapies in Health and Medicine, 19, 26-30.

[29] Asadi, N., Maharlouei, N., Khalili, A., Darabi, Y., Davoodi, S., Shahraki, H.R., et al. (2015) Effects of LI-4 and SP-6 Acupuncture on Labor Pain, Cortisol Level, and Duration of Labor. Journal of Acupuncture and Meridian Studies, 8, 249-254. https://doi.org/10.1016/j.jams.2015.08.003

[30] Hamidzadeh, A., Shahpourian, F., Orak, R.J., Montazeri, A.S. and Khosravi, A. (2012) Effects of LI4 Acupressure on Labor Pain in the First Stage of Labor. Journal of Midwifery \& Women's Health, 57, 133-138. https://doi.org/10.1111/j.1542-2011.2011.00138.x

[31] Chaillet, N., Belaid, L., Crochetière, C., Roy, L., Gagné, G.P., Moutquin, J.M., et al. (2014) Nonpharmacologic Approaches for Pain Management during Labor Compared with Usual Care: A Meta-Analysis. Birth, 41, 122-137. https://doi.org/10.1111/birt.12103

[32] Borup, L., Wurlitzer, W., Hedegaard, M., Kesmodel, U.S. and Hvidman, L. (2009) Acupuncture as Pain Relief during Delivery: A Randomized Controlled Trial. Birth, 36, 5-12. https://doi.org/10.1111/j.1523-536X.2008.00290.x

[33] Chung, U., Hung, L.C., Kuo, S.C. and Huang, C.L. (2003) Effects of LI4 and BL67 Acupressure on Labor Pain and Uterine Contractions in the First Stage of Labour. Journal of Nursing Research, 11, 251-260. https://doi.org/10.1097/01.JNR.0000347644.35251.c1

[34] Hjelmstedt, A., Shenoy, S.T., Stener-Victorin, E., Lekander, M., Bhat, M., Balakumaran, L., et al. (2010) Acupressure to Reduce Labor Pain: A Randomized Controlled Trial. Acta Obstetricia et Gynecologica Scandinavica, 89, 1453-1459.

https://doi.org/10.3109/00016349.2010.514323

[35] Nesheim, B.-I., Kinge, R., Berg, B., Alfredsson, B., Allgot, E., Hove, G., et al. (2003) Acupuncture during Labor Can Reduce the Use of Meperidine: A Controlled Clinical Study. Clinical Journal of Pain, 19, 187-191. https://doi.org/10.1097/00002508-200305000-00006

[36] Ziaei, S. and Hajipour, L. (2006) Effect of Acupuncture on Labor. International Journal of Gynaecology and Obstetrics, 92, 71-72. https://doi.org/10.1016/j.ijgo.2005.09.008

[37] MacKenzie, I.Z., Xu, J., Cusick, C., Midwinter-Morten, H., Meacher, H., Mollison, J., et al. (2011) Acupuncture for Pain Relief during Induced Labour in Nulliparae: A Randomised Controlled Study. BJOG, 118, 440-447. https://doi.org/10.1111/j.1471-0528.2010.02825.x 
[38] Ma, W., Bai, W., Lin, C., Zhou, P., Xia, L., Zhao, C., et al. (2011) Effects of Sanyinjiao (SP6) with Electroacupuncture on Labour Pain in Women during Labour. Complementary Therapies in Medicine, 19, S13-S18. https://doi.org/10.1016/j.ctim.2010.09.001

[39] Labrecque, M., Nouwen, A., Bergeron, M. and Rancourt J.F. (1999) A Randomized Controlled Trial of Nonpharmacologic Approaches for Relief of Low Back Pain During Labor. Journal of Family Practice, 48, 259-263.

[40] Van Der Spark, J. (2000) Pain Relief in Labour by Transcutaneous Electrical Nerve Stimulation. Archives of Gynecology and Obstetrics, 264, 131-136. https://doi.org/10.1007/s004040000099

[41] Smith, C.A., Collins, C.T., Crowther, C.A. and Levett, K.M. (2011) Acupuncture or Acupressure for Pain Management in Labour. Cochrane Database of Systematic Reviews, 8, CD009232.

[42] Hamidzadeh, A., Shahpourian, F., Orak, R.J., Montazeri, A.S. and Khosravi, A. (2012) Effects of LI4 Acupressure on Labor Pain in the First Stage of Labor. Journal of Midwifery \& Women's Health, 57, 133-138. https://doi.org/10.1111/j.1542-2011.2011.00138.x

[43] Huang, T., Yang, Y. and Huang, X. (2008) Selection of Acupoints and Opportunity for Acupuncture Analgesia in Delivery. Journal of Traditional Chinese Medicine, 49, 625-628.

[44] Kashanian, M. and Shahali, S. (2010) Effects of Acupressure at the Sanyinjiao Point (SP6) on the Process of Active Phase of Labor in Nulliparas Women. Journal of Maternal-Fetal \& Neonatal Medicine, 23, 638-641. https://doi.org/10.3109/14767050903277662

[45] Lee, M.K., Chang, S.B. and Kang, D.K. (2004) Effects of SP6 Acupressure on Laborpain and Length of Delivery Time in Women during Labor. Journal of Alternative and Complementary Medicine, 10, 959-965. https://doi.org/10.1089/acm.2004.10.959

[46] Martensson, L., Stener-Victorin, E. and Wallin, G. (2008) Acupuncture versus Subcutaneous Injections of Sterile Water as Treatment for Labour Pain. Acta Obstetricia et Gynecologica Scandinavica, 87, 171-177. https://doi.org/10.1080/00016340701797799

[47] Qu, F. and Zhou, J. (2007) Electro-Acupuncture in Relieving Labor Pain. Evidence-Based Complementary and Alternative Medicine, 4, 125-130. https://doi.org/10.1093/ecam/nel053

[48] Ramnerö, A., Hanson, U. and Kihlgren, M. (2002) Acupuncture Treatment during Labour-A Randomised Controlled Trial. BJOG, 109, 637-644.

[49] Skilnand, E., Fossen, D. and Heiberg, E. (2002) Acupuncture in the Management of Pain in Labor. Acta Obstetricia et Gynecologica Scandinavica, 81, 943-948. https://doi.org/10.1034/j.1600-0412.2002.811008.x

[50] Cho, S.H., Lee, H. and Ernst, E. (2010) Acupuncture for Pain Relief in Labour: A Systematic Review and Meta-Analysis. BJOG, 117, 907-920. https://doi.org/10.1111/j.1471-0528.2010.02570.x

[51] Zhang, Y. (2006) Clinical Observation on Therapeutic Effect of Electroacupuncture on SP6 for Labor Pain. Beijing University of Chinese Medicine, Beijing, 42.

[52] Zhou, P. (2007) Clinical Observation on Relieving the Labor Pain of Electroacupuncture on SP6. Beijing University of Chinese Medicine, Beijing, 43.

[53] Lee, H. and Ernst, E. (2004) Acupuncture for Labor Pain Management: A Systematic Review. American Journal of Obstetrics and Gynecology, 191, 1573-1579. https://doi.org/10.1016/j.ajog.2004.05.027

[54] Huntley, A.L., Coon, J.T. and Ernst, E. (2004) Complementary and Alternative Medicine for Labor Pain: A Systematic Review. American Journal of Obstetrics and Gynecology, 191, 36-44. https://doi.org/10.1016/j.ajog.2003.12.008 
[55] Dettori, J. (2010) The Random Allocation Process: Two Things You Need to Know. Evidence-Based Spine-Care Journal, 1, 7-9. https://doi.org/10.1055/s-0030-1267062

[56] Arendt, K.W. and Tessmer-Tuck, J.A. (2013) Nonpharmacologic Labor Analgesia. Clinics in Perinatology, 40, 351-371. https://doi.org/10.1016/j.clp.2013.05.007

[57] Hanley, G.E., Munro, S., Greyson, D., Gross, M.M., Hundley, V., Spiby, H., et al. (2016) Diagnosing Onset of Labor: A Systematic Review of Definitions in the Research Literature. BMC Pregnancy and Childbirth, 16, 71. https://doi.org/10.1186/s12884-016-0857-4

\section{Submit or recommend next manuscript to SCIRP and we will provide best service} for you:

Accepting pre-submission inquiries through Email, Facebook, LinkedIn, Twitter, etc.

A wide selection of journals (inclusive of 9 subjects, more than 200 journals)

Providing 24-hour high-quality service

User-friendly online submission system

Fair and swift peer-review system

Efficient typesetting and proofreading procedure

Display of the result of downloads and visits, as well as the number of cited articles

Maximum dissemination of your research work

Submit your manuscript at: http://papersubmission.scirp.org/

Or contact cm@scirp.org 